\title{
Stability and Multiscroll Attractors of Control Systems via the Abscissa
}

\author{
Edgar-Cristian Díaz-González, ${ }^{1}$ Baltazar Aguirre-Hernández, ${ }^{1}$ \\ Jorge Antonio López-Rentería, ${ }^{2}$ Eric Campos-Cantón, ${ }^{3,4}$ \\ and Carlos Arturo Loredo-Villalobos ${ }^{3}$ \\ ${ }^{1}$ Departamento de Matemáticas, Universidad Autónoma Metropolitana Iztapalapa, San Rafael Atlixco 186, 09340 CDMX, Mexico \\ ${ }^{2}$ Departamento de Matemáticas, Universidad de Sonora, Hermosillo, SON, Mexico \\ ${ }^{3}$ División de Matemáticas Aplicadas, IPICyT, 78216 San Luis, SLP, Mexico \\ ${ }^{4}$ Mathematics Department, University of Houston, Houston, TX 77204-3008, USA
}

Correspondence should be addressed to Carlos Arturo Loredo-Villalobos; calv@xanum.uam.mx

Received 4 January 2017; Revised 9 May 2017; Accepted 12 July 2017; Published 17 August 2017

Academic Editor: Driss Mehdi

Copyright (c) 2017 Edgar-Cristian Díaz-González et al. This is an open access article distributed under the Creative Commons Attribution License, which permits unrestricted use, distribution, and reproduction in any medium, provided the original work is properly cited.

\begin{abstract}
We present an approach to generate multiscroll attractors via destabilization of piecewise linear systems based on Hurwitz matrix in this paper. First we present some results about the abscissa of stability of characteristic polynomials from linear differential equations systems; that is, we consider Hurwitz polynomials. The starting point is the Gauss-Lucas theorem, we provide lower bounds for Hurwitz polynomials, and by successively decreasing the order of the derivative of the Hurwitz polynomial one obtains a sequence of lower bounds. The results are extended in a straightforward way to interval polynomials; then we apply the abscissa as a measure to destabilize Hurwitz polynomial for the generation of a family of multiscroll attractors based on a class of unstable dissipative systems (UDS) of affine linear type.
\end{abstract}

\section{Introduction}

Consider the parametric dynamical system

$$
\dot{\mathbf{x}}=f(\mathbf{x}, \mu),
$$

where $\mathbf{x} \in \mathbb{R}^{n}$ is the state vector, $\mu \in \mathbb{R}^{m}$ is a parameter vector, and $f$ is an enough smooth vector field. Several techniques have been proposed in the analysis of the solutions behavior of a dynamical system. The Hartman-Grobman theorem establishes that its internal evolution is determined by its Jacobian matrix. That is, the behavior of its solutions is described by the spectrum of its linearization. If all of the solutions of a dynamical system converge to an equilibrium point then it is said to be a locally asymptotically stable system. The importance of studying Hurwitz polynomials is due to its usefulness in the stability analysis of linear systems: if the characteristic polynomial of a linearized system is Hurwitz (roots with negative real part) then it is asymptotically stable. This has motivated researchers working on applications seeking such polynomials. Maxwell [1] posed the problem in the following way: How can one find the necessary and sufficient conditions to decide whether a polynomial has all its roots with negative real part? A solution was given by Hurwitz [2] and it is known as the Routh-Hurwitz criterion. Related information about Hurwitz polynomials can be found in [36].

The study of stability with a polynomial approach had an important impulse when Kharitonov's theorem was published in 1978. This theorem gives conditions for the stability of an interval family of polynomials (see [7]). Since then, a lot of works related to this theorem have been published (see, e.g., [8-12]). The importance of studying the stability of families of polynomials can be appreciated in applications where the presence of uncertainties in the polynomial 
coefficients has to be taken into account. Other families of polynomials that have been investigated are the segments of polynomials (see [13-16]). Good references on families of stable polynomials are [3, 17-19]. The importance of knowing the abscissa of stability has been pointed out in [20-22]. Lower bounds were reported in $[23,24]$; these are the first works about the abscissa of stability; and upper bounds were obtained in Bialas [25], Henrici [26], and Olifirov [27].

However, stability is not always required. For example, there is a class of chaotic dynamical systems based on unstable equilibria. Several times a structural change is given by one bifurcation parameter of $\mu$ that generates bifurcation in the solutions of the system. Generating chaotic behavior is the subject of interest in several areas in mathematics and engineering insomuch that researchers have taken the task of design systems with diverse techniques undergoing chaotic behavior with and without equilibria. One of the different chaotic behaviors is the presence of multiscroll attractor. Good references where the generation of multiscrolls has been studied are the works [28-35]. In this paper we use the abscissa of stability of Hurwitz polynomials to study the stability of systems in order to generate multiscroll attractors. To achieve the design of a chaotic system, a technique involving lower bounds for stabilizing and breaking down the stability to make multiscroll attractors arise is described. The rest of the paper is organized as follows: In Section 2, basic definitions and results needed for the development of our technique are given. In Section 3, the relation between the abscissas of stability $\sigma_{p}$ and $\sigma_{p}^{\prime}$, of a Hurwitz polynomial $p(t)$ and its derivative polynomial $p^{\prime}(t)$, respectively, is studied. Therein the relationship is the following inequality $\sigma_{p}^{\prime}<\sigma_{p}$ which is used to obtain a lower bound for the abscissa of stability of a polynomial or an interval family of Hurwitz polynomials. We use the Gauss-Lucas Theorem 2 to analyze the Hurwitz stability of a polynomial and its derivative. Finally, in Section 4 an application of the lower bound to generate chaos is given.

\section{Preliminaries}

Consider an asymptotically stable linear system given by

$$
\dot{\mathbf{x}}=A \mathbf{x}
$$

where $\mathbf{x}$ is the state vector of the system and $A \in \mathbb{R}^{n \times n}$ is a linear operator. Let $p(t)$ be the characteristic polynomial of $A$. The abscissa of stability of polynomial $p(t)$ is given by the following definition.

Definition 1. If $p(t)$ is a Hurwitz polynomial and $z_{1}, z_{2}, \ldots, z_{n}$ are its zeros then $\sigma_{p}$ the abscissa of stability of $p(t)$ is defined by

$$
\sigma_{p}=\max _{1 \leq i \leq n}\left\{\operatorname{Re} z_{i}\right\}
$$

If $\underline{\sigma}_{p}$ and $\bar{\sigma}_{p}$ are numbers such that $\underline{\sigma}_{p} \leq \sigma_{p} \leq \bar{\sigma}_{p}$, then they are named lower and upper bound, respectively.
In Section 4 we consider a polynomial $p(t-r)$, so that, varying the parameter $r$, then we get destabilization of the polynomial $p(t)$ and we get the generation of multiscroll. In Section 4 we give the details. Now we present a useful theorem in our results.

Theorem 2 (Gauss-Lucas [36]). Let $K$ be any convex polygon enclosing all the zeros of the polynomial $f(z)$. Then the zeros of $f^{\prime}(z)$ lie in $K$.

Remark 3. Let us recall that a set of points is convex if it contains, with any two points $P, Q$ in the set, the line segment joining $P$ and $Q$.

The abscissa of stability $\sigma_{p}$ of the characteristic polynomial of system (2) gives certain minimum rate of decay. Zakian and Al-Naib indicated that in computer-aided design of dynamical and control systems the numerical computation of the abscissa of stability is required (see [21, 37-39]) to warrant stability under perturbations.

\section{Main Results}

3.1. Abscissa of Hurwitz Polynomials: An Inequality between $\sigma_{p}$ and $\sigma_{p^{\prime}}$. Consider the polynomial $p(t)=a_{n} t^{n}+a_{n-1} t^{n-1}+$ $\cdots+a_{1} t+a_{0}$ with $a_{i} \in \mathbb{R}$ for all $i=0, \ldots, n$.

Theorem 4. If $p(t)=a_{n} t^{n}+a_{n-1} t^{n-1}+\cdots+a_{1} t+a_{0}$ is a Hurwitz polynomial $(n \geq 2)$ and $\sigma_{p}$ and $\sigma_{p^{\prime}}$ are the abscissas of stability of $p$ and $p^{\prime}=d p / d t$, respectively, then $\sigma_{p^{\prime}} \leq \sigma_{p}$.

Proof. Let $p(t)$ be a Hurwitz polynomial. If $\xi_{1}, \xi_{2}, \ldots, \xi_{n}$ are the roots of $p(t)$ then its abscissa of stability $\sigma_{p}$ is given by $\sigma_{p}=-R$, where $R=\max \left\{\bar{r}>0: \xi_{1}+r, \xi_{2}+r, \ldots, \xi_{n}+\right.$ $\left.r \in \mathbb{C}^{-}, \forall r<\bar{r}\right\}$. Then $\sigma_{p}=-R$, where $R=\max \{\bar{r}>$ 0 : $p(t-r)$ is a Hurwitz polynomial, $\forall r<\bar{r}$ \}. Now, by the Gauss-Lucas Theorem 2 if $p(z)$ is Hurwitz then $p^{\prime}(t)$ is Hurwitz. Consequently, if $p(t-r)$ is a Hurwitz polynomial then $p^{\prime}(t-r)$ is a Hurwitz polynomial. This implies that $\sigma_{p^{\prime}} \leq \sigma_{p}$, as we claim.

Example 5. Consider the polynomial $p(t)=t^{3}+(19 / 6) t^{2}+$ $(8 / 3) t+2 / 3$. The abscissa of stability of $p(t)$ is $\sigma_{p}=-0.5$ and the abscissa of stability of $p^{\prime}(t)=3 t^{2}+(19 / 3) t+8 / 3$ is $\sigma_{p^{\prime}} \approx-0.58$. We see that $\sigma_{p^{\prime}}<\sigma_{p}$.

Example 6. Consider $p(t)=t^{4}+(25 / 6) t^{3}+(35 / 6) t^{2}+(10 / 3) t+$ $2 / 3$. The abscissa of stability of $p(t)$ is $\sigma_{p}=-0.5$ and the abscissa of stability of $p^{\prime}(t)=4 t^{3}+(25 / 2) t^{2}+(35 / 3) t+10 / 3$ is $\sigma_{p^{\prime}} \approx-0.57$. Therefore $\sigma_{p^{\prime}}<\sigma_{p}$.

Example 7. Let $p(t)=t^{3}+4 t^{2}+5 t+2$. The abscissa of stability of $p(t)$ is $\sigma_{p}=-1$ and the abscissa of stability of $p^{\prime}(t)=3 t^{2}+$ $8 t+5$ is $\sigma_{p^{\prime}}=-1$. In this case we have that $\sigma_{p^{\prime}}=\sigma_{p}$.

Remark 8. Theorem 4 leads to glimpsing the following open problem: if $p(t)$ is a Hurwitz polynomial, find necessary and sufficient conditions to make the equality $\sigma_{p^{\prime}}=\sigma_{p}$ hold. 


\subsection{A Lower Bound of the Abscissa of Stability of a Polynomial}

Theorem 9. Let $p(t)=a_{n} t^{n}+a_{n-1} t^{n-1}+a_{n-2} t^{n-2}+\cdots+a_{2} t^{2}+$ $a_{1} t+a_{0}$ be a Hurwitz polynomial with positive coefficients and denote $\Delta_{p}=\left[2(n-1) a_{n-1}\right]^{2}-8 n(n-1) a_{n} a_{n-2}$. The following inequalities hold:

(a) If $\Delta_{p} \geq 0$, then

$$
\frac{-2(n-1) a_{n-1}+\sqrt{\Delta_{p}}}{2 n(n-1) a_{n}} \leq \sigma_{p}
$$

(b) If $\Delta_{p}<0$, then $-a_{n-1} / n a_{n} \leq \sigma_{p}$.

Proof. If $p(t)=a_{n} t^{n}+a_{n-1} t^{n-1}+a_{n-2} t^{n-2}+\cdots+a_{1} t+a_{0}$ is a Hurwitz polynomial then $p^{(n-2)}(t)=n(n-1) \cdots 3 a_{n} t^{2}+(n-$ 1) $(n-2) \cdots 2 a_{n-1} t+(n-2) \cdots 2 a_{n-2}$ is a Hurwitz polynomial. By Theorem 4 we have that $\sigma_{p^{(n-2)}} \leq \sigma_{p^{(n-3)}} \leq \cdots \leq \sigma_{p^{\prime}} \leq \sigma_{p}$. But $p^{(n-2)}(t)=0$ if and only if $n(n-1) a_{n} t^{2}+2(n-1) a_{n-1} t+$ $2 a_{n-2}=0$. If $\Delta_{p} \geq 0$, then

$$
\frac{-2(n-1) a_{n-1}+\sqrt{\Delta_{p}}}{2 n(n-1) a_{n}}=\sigma_{p^{(n-2)}} \leq \sigma_{p}
$$

and (a) is established. The proof of (b) follows in the same way.

Example 10. For the polynomial $p(t)=6 t^{5}+43 t^{4}+110 t^{3}+$ $125 t^{2}+64 t+12$ we have that $n=5, a_{n-2}=110, a_{n-1}=43$, $a_{n}=6$, and $\Delta_{p}=12736 \geq 0$. By part (a) of Theorem 9 we have that

$$
\frac{-2(n-1) a_{n-1}+\sqrt{\Delta_{p}}}{2 n(n-1) a_{n}}
$$

is a lower bound of $\sigma_{p}$; that is, $-0.96 \leq-1 / 2=\sigma_{p}$.

Example 11. Consider $p(t)=t^{4}+3 t^{3}+5 t^{2}+4 t+2$. Here $n=4$, $a_{n-2}=5, a_{n-1}=3, a_{n}=1$, and $\Delta_{p}=-156 \leq 0$. By part (b) of Theorem 9 we have that $-a_{n-1} / n a_{n}$ is a lower bound of $\sigma_{p}$; that is, $-3 / 4 \leq-1 / 2=\sigma_{p}$.

Remark 12. Consider

$$
\begin{aligned}
S_{m i}=-\left[\left(\begin{array}{l}
n \\
m
\end{array}\right)^{-1}\left(\begin{array}{l}
n \\
i
\end{array}\right)\left(\frac{a_{m}}{a_{i}}\right)\right]^{1 /(i-m)} & \text { for } m=0,1, \ldots, i-1, i=1, \ldots, n .
\end{aligned}
$$

Note that (7) is a set of lower bounds that were obtained in $[23,24]$. The bound obtained in Theorem 9 (b) $-a_{n-1} / n a_{n}$ is in the set of lower bounds given in (7): taking $m=n-1$ and $i=n$ we can see that $S_{(n-1) n}=-a_{n-1} / n a_{n}$.

In fact, another way to obtain $S_{(n-1) n}$ is by mean of the abscissa of stability of the $(n-1)$ th derivative $p^{(n-1)}(t)=$ $n(n-1) \cdots 2 a_{n} t+(n-1)(n-2) \cdots 2 a_{n-1}$. Note that Theorem 9(a) is a new lower bound for the abscissa of stability and since it depends on three coefficients of $p(t)$ while the lower bounds in (7) only depend on two coefficients of $p(t)$, the bound in Theorem 9(a) is in some cases better than the bound in Theorem 9(b) as is illustrated by the following example.

Example 13. Consider the following polynomial $p(t)=6 t^{5}+$ $43 t^{4}+110 t^{3}+125 t^{2}+64 t+12$. Here $n=5, a_{n-2}=110$, $a_{n-1}=43$, and $a_{n}=6$. By item (a) from Theorem 9 we have that

$$
\frac{-2(n-1) a_{n-1}+\sqrt{\Delta_{p}}}{2 n(n-1) a_{n}} \approx-0.96
$$

is a lower bound of $\sigma_{p}=-1 / 2$ and $-a_{n-1} / n a_{n}=-1.43<$ $-0.96<-1 / 2=\sigma_{p}$.

Example 14. Let $p(t)=6 t^{3}+19 t^{2}+16 t+4$. Here $n=3$, $a_{n-2}=16, a_{n-1}=19$, and $a_{n}=6$. By item (a) from Theorem 9 we have that

$$
\frac{-2(n-1) a_{n-1}+\sqrt{\Delta_{p}}}{2 n(n-1) a_{n}} \approx-0.58
$$

is a lower bound of $\sigma_{p}=-1 / 2$ and $-a_{n-1} / n a_{n}<-0.58<\sigma_{p}$.

3.3. Lower Bounds for the Abscissa of Stability of an Interval Family of Hurwitz Polynomials. For a family of Hurwitz polynomials of degree $n$ of the form

$$
\begin{aligned}
\mathscr{F} & =\left\{f(t): f(t)=\sum_{j=0}^{n} a_{n-j} t^{n-j}, a_{i} \in\left[\alpha_{i}, \beta_{i}\right], i\right. \\
& =0,1, \ldots, n\}
\end{aligned}
$$

the abscissa of stability is defined by $\max _{p \in \mathscr{F}} \sigma_{p}$.

Theorem 15. Consider the family of Hurwitz polynomials $f(t)=a_{n} t^{n}+a_{n-1} t^{n-1}+\cdots+a_{1} t+a_{0}$ with $0<\alpha_{j} \leq a_{j} \leq \beta_{j}$, $j=0,1, \ldots, n$; we have that

(a) $-\beta_{n-1} /\left(n \alpha_{n}\right)$ is a lower bound for the abscissa of stability of the family of polynomials;

(b) if $\left[2(n-1) \alpha_{n-1}\right]^{2}-8 n(n-1) \beta_{n} \beta_{n-2} \geq 0$, then $-\beta_{n-1} / n \alpha_{n}$ and

$$
\frac{-2(n-1) \beta_{n-1}+\sqrt{\left[2(n-1) \alpha_{n-1}\right]^{2}-8 n(n-1) \beta_{n} \beta_{n-2}}}{2 n(n-1) \beta_{n}}
$$

are lower bounds for the abscissa of stability of the family of polynomials.

Proof. From item (b) of Theorem 9, $-a_{n-1} /\left(n a_{n}\right)$ is a lower bound for the abscissa of stability of $f(t)=a_{n} t^{n}+a_{n-1} t^{n-1}+$ 
$\cdots+a_{1} t+a_{0}$. On the other hand, since $-\beta_{n-1} \leq-a_{n-1} \leq$ $-\alpha_{n-1}$ and $1 / \beta_{n} \leq 1 / a_{n} \leq 1 / \alpha_{n}$, we have that $-\beta_{n-1} / n \alpha_{n} \leq$ $-a_{n-1} / n a_{n}$.

For item (b) of Theorem 9 suppose that $\left[2(n-1) \alpha_{n-1}\right]^{2}-$ $8 n(n-1) \beta_{n} \beta_{n-2} \geq 0$. From

$$
\begin{aligned}
\alpha_{n-2} & \leq a_{n-2} \leq \beta_{n-2}, \\
\alpha_{n-1} & \leq a_{n-1} \leq \beta_{n-1}, \\
\alpha_{n} & \leq a_{n} \leq \beta_{n},
\end{aligned}
$$

the next inequalities are obtained:

(1) $2(n-1) \alpha_{n-1} \leq 2(n-1) a_{n-1} \leq 2(n-1) \beta_{n-1}$,

(2) $8 n(n-1) \alpha_{n} \alpha_{n-2} \leq 8 n(n-1) a_{n} a_{n-2} \leq 8 n(n-1) \beta_{n} \beta_{n-2}$,

(3) $1 / \beta_{n} \leq 1 / a_{n} \leq 1 / \alpha_{n}$,

(4) $-2(n-1) \beta_{n-1} \leq-2(n-1) a_{n-1}$,

(5) $\left[2(n-1) \alpha_{n-1}\right]^{2}-8 n(n-1) \beta_{n} \beta_{n-2} \leq\left[2(n-1) a_{n-1}\right]^{2}-$ $8 n(n-1) a_{n} a_{n-2}$,

(6) $1 / 2 n(n-1) \beta_{n} \leq 1 / 2 n(n-1) a_{n}$.

Thus

$$
\begin{aligned}
& \frac{-2(n-1) \beta_{n-1}+\sqrt{\left[2(n-1) \alpha_{n-1}\right]^{2}-8 n(n-1) \beta_{n} \beta_{n-2}}}{2 n(n-1) \beta_{n}} \\
& \leq \frac{-2(n-1) a_{n-1}+\sqrt{\Delta_{p}}}{2 n(n-1) a_{n}} .
\end{aligned}
$$

This proves Theorem 15.

Remark 16. Note that for every interval family of Hurwitz polynomials we give the lower bound $-\beta_{n-1} /\left(n \alpha_{n}\right)$. If additionally the family satisfies $\left[2(n-1) \alpha_{n-1}\right]^{2}-8 n(n-1) \beta_{n} \beta_{n-2} \geq$ 0 then we can give a second lower bound given by

$$
\frac{-2(n-1) \beta_{n-1}+\sqrt{\left[2(n-1) \alpha_{n-1}\right]^{2}-8 n(n-1) \beta_{n} \beta_{n-2}}}{2 n(n-1) \beta_{n}} .
$$

Remark 17. In Theorem 15 we have two lower bounds, but there could be more lower bounds. The abscissa is the maximum of all of them. That is, another way of obtaining the abscissa of stability is to take the maximum of the lower bounds.

Example 18. Consider the family of Hurwitz polynomials

$$
f(t)=a_{4} t^{4}+a_{3} t^{3}+a_{2} t^{2}+a_{1} t+a_{0},
$$

where $10 \leq a_{0} \leq 20,23 \leq a_{1} \leq 34,18 \leq a_{2} \leq 19,5 \leq a_{3} \leq 7$, and $1 \leq a_{4} \leq 1$. Here $\alpha_{0}=10, \beta_{0}=20, \ldots, \alpha_{4}=1, \beta_{4}=1$, and $n=4$.

Since $\left[2(n-1) \alpha_{n-1}\right]^{2}-8 n(n-1) \beta_{n} \beta_{n-2}=-828<0$ by part (a) of Theorem 15 we have that $-\beta_{n-1} / n \alpha_{n}=-\beta_{3} / 4 \alpha_{4}=$ $-7 / 4$ is a lower bound of the abscissa of stability of the family Hurwitz polynomials.
Example 19. Consider the family of Hurwitz polynomials

$$
f(t)=a_{3} t^{3}+a_{2} t^{2}+a_{1} t+a_{0}
$$

where $0.25 \leq a_{0} \leq 1.25,0.75 \leq a_{1} \leq 1.25,2.75 \leq a_{2} \leq 3.25$, and $0.25 \leq a_{3} \leq 1.75$. Here $\alpha_{0}=0.25, \beta_{0}=1.25, \ldots, \alpha_{3}=$ $0.25, \beta_{3}=1.75$, and $n=3$. Since $\left[2(n-1) \alpha_{n-1}\right]^{2}-8 n(n-$ 1) $\beta_{n} \beta_{n-2}=46>0$, by item (b) from Theorem 15 we have that

$$
\frac{-2(n-1) \beta_{n-1}+\sqrt{\left[2(n-1) \alpha_{n-1}\right]^{2}-8 n(n-1) \beta_{n} \beta_{n-2}}}{2 n(n-1) \beta_{n}}
$$

$$
\approx-0.41
$$

which is a lower bound of the abscissa of stability of the family of Hurwitz polynomials.

\section{The Abscissa to Generate Instability and Multiscrolls Attractors}

In the study of multiscroll attractors different aspects are interesting and one of them is when the multiscroll attractor exists for a particular set of system's parameters; then the interest is about robustness against parametric perturbation. For instance, we would like to know the variation of the values of parameters of a given system in order to preserve the multiscroll attractor. In this direction a polynomial approach has been used to find the maximal robust dynamics [40] and for studying the maximum range for a set of parameters to preserve the useful instability for the generation of multiscroll attractors [41]. Now, let us apply the abscissa approach for finding the lower bound of the abscissa of hyperbolicity and instability needed in UDS to generate multiscroll attractors. The linear system (2) under a control action is given as follows:

$$
\dot{x}=A x+B u,
$$

with Hurwitz characteristic polynomial of $A, p(t)=t^{n}+$ $a_{n-1} t^{n-1}+\cdots+a_{1} t+a_{0}$. Define $f_{r}(t)=p(t-r)$, with $r \geq 0$. Note that $f_{r}(t)$ is a set of polynomials such that $f_{0}(t)=p(t)$ is a Hurwitz polynomial and the abscissa of stability can be calculated by

$$
\sigma_{f_{r}}=-\max _{\bar{r}}\left\{\bar{r}>0 \mid f_{r}(t) \text { is Hurwitz } \forall r, r<\bar{r}\right\} .
$$

Now, by Taylor's theorem $f_{r}(t)=p(t-r)$ can be rewritten as

$$
\begin{aligned}
f_{r}(t) & =t^{n}+\frac{p^{(n-1)}(-r)}{(n-1) !} t^{n-1}+\cdots+\frac{p^{\prime}(-r)}{1 !} t+p(-r) \\
& =t^{n}+A_{n-1}(r) t^{n-1}+\cdots+A_{1}(r) t+A_{0}(r) .
\end{aligned}
$$

If $r=-\sigma_{p}$ then $f_{r}(t)$ has roots in the imaginary axis. Thence, the system is unstable in the interval $\left(-\sigma_{p}, \infty\right)$. Let us describe the class of instabilities by considering the following system in $\mathbb{R}^{3}$. 
Definition 20. We have the following system:

$$
\dot{x}=A x,
$$

where $x \in R^{3}$ is the state vector, $A \in R^{3 \times 3}$ is a linear operator with eigenvalues $\lambda_{i}$, and $i=1,2,3$ is said to be dissipative if $\sum_{i=1}^{3} \lambda_{i}<0$. The system is said to be unstable and dissipative of type I (UDS-I) if one of its eigenvalues is a negative real number and the other two are complex conjugate numbers with positive real part; and it is said to be of type II (UDS-II) if one of its eigenvalues is a positive real number and the other two are complex conjugate numbers with negative real part.

This work is based on UDS-I, so a generalization of the above definition for UDS-I with dimension greater than three can be given as follows.

Definition 21. The system given by (21) where $x \in \mathbb{R}^{n}$, $A \in \mathbb{R}^{n \times n}$, and eigenvalues $\lambda_{i}, i=1,2, \ldots, n$, is said to be dissipative if $\sum_{i=1}^{n} \lambda_{i}<0$. The system is said to be unstable and dissipative of type I (UDS-I) if $n-2$ of its eigenvalues are negative real numbers and the other two are complex conjugate numbers with positive real part.

Due to the relation between the linear system like (21) and its characteristic polynomial, we shall say that an $n$ degree polynomial $p(t)$ is dissipative if the sum of its roots is negative. In a similar way, $p(t)$ will be a UDS-I polynomial if its roots satisfy Definition 21 for systems of type I. Notice that Definition 21 is only one possibility to define UDS considering $n-2$ negative real numbers.

Lemma 22. Let $p(t)$ be a real $n$-degree Hurwitz polynomial with roots $t_{1}, \ldots, t_{n}$. If $f_{r}(t)=p(t-r)$ is unstable and dissipative, then the following conditions are satisfied:

$$
\begin{aligned}
& \text { (i) } r>-\sigma_{p} . \\
& \text { (ii) } r<U_{d i s s(p)}=-(1 / n) \sum_{j=1}^{n} t_{j} .
\end{aligned}
$$

Proof. The proof of (i) is obvious. We will focus on the proof of (ii). Firstly, it is not too hard to see that if the root $t_{j}$ of $p(t)$ has nonzero imaginary part, then its translation $r+t_{j}$ and its conjugate are roots of $f_{r}(t)$, with $r \in \mathbb{R}$. Namely, by writing $p(t)=\prod_{j=1}^{n}\left(t-t_{j}\right)$, then

$$
f_{r}(t)=\prod_{j=1}^{n}\left[t-r-t_{j}\right]=\prod_{j=1}^{n}\left[t-\left(r+t_{j}\right)\right] .
$$

Thence,

$$
\sum_{j=1}^{n}\left(r+t_{j}\right)=\sum_{j=1}^{n} t_{j}+n r
$$

and since $\sum_{j=1}^{n} t_{j}<0$, then $-(1 / n) \sum_{j=1}^{n} t_{j}>0$ and

$$
\begin{aligned}
\sum_{j=1}^{n} t_{j}+n r & <0 \Longleftrightarrow \\
r & \Longleftrightarrow-\frac{1}{n} \sum_{j=1}^{n} t_{j} .
\end{aligned}
$$

Therefore, if $f_{r}(t)$ is unstable and dissipative, then $r<$ $-(1 / n) \sum_{j=1}^{n} t_{j}$, as we claim.

Remark 23. The previous lemma provides an upper bound for dissipativity. However, it may happen that $-\sigma_{p}=U_{\text {diss }(p)}$ in the case when $\operatorname{Re}\left(t_{j}\right)=c$, for all $j=1, \ldots, n$.

Given the fact that a Hurwitz polynomial $p(t)$ can be perturbed to be unstable for $\left(\sigma_{p}, \infty\right)$ and that $U_{\operatorname{diss}(p)}$ is an upper bound for the dissipativity, it is possible to carry the system from stability to instability in the sense of UDS if at least one of its roots has different real part than the others. The following result is immediate from the aforementioned discussion.

Corollary 24. Consider the Hurwitz polynomial $p(t)=$ $\prod_{j=1}^{n}\left(t-t_{j}\right)$, with $n-2$ real roots and a pair of conjugate complex roots, say, $t_{i}, t_{i+1}$, for some $1 \leq i \leq n$. Then

(i) $f_{r}(t)$ is Hurwitz if and only if $r<-\sigma_{p}$.

(ii) If $\operatorname{Re}\left(t_{i}\right) \neq t_{j}, i \neq j$, then $f_{r}(t)$ is UDS if and only if $r \in\left(-\sigma_{p}, U_{\text {diss }(p)}\right)$.

In order to generate multiscroll attractors, let us consider the control system

$$
\dot{\mathbf{x}}=\mathbf{A} \mathbf{x}+\mathbf{B} S+b u
$$

where $\mathbf{x}=\left[x_{1}, x_{2}, \ldots, x_{n}\right]^{T} \in \mathbb{R}^{n}$ is the state vector, $\mathbf{B} \in \mathbb{R}^{n}$ stands for a real affine vector, and $\mathbf{A}=\left[a_{i j}\right] \in \mathbb{R}^{n \times n}$ with $i, j=1,2, \ldots, n$ denotes a nonsingular linear matrix.

Let $p_{A}(t)$ be the characteristic polynomial of the system, $b^{T}=(0,0, \ldots, 0,1)$, and $S$ is the following step function:

$$
S= \begin{cases}s_{1} & \text { for } c_{1}<x_{1} \\ s_{2} & \text { for } c_{2}<x_{1} \leq c_{1} \\ \vdots & \\ s_{m} & \text { for } c_{m}<x_{1} \leq c_{m-1}\end{cases}
$$

where the values $c_{i}$ 's must be chosen in a suitable way that will be explained below. Define the linear control $u=c^{T}(r) x=$ $\left(a_{0}-A_{0}(r), a_{1}-A_{1}(r), \ldots, a_{n-1}-A_{n-1}(r)\right) x$, where $A_{j}(r)=$ $p^{j}(-r) / j$. Then the controlled system is

$\dot{\mathbf{x}}$

$$
\begin{aligned}
= & \left(\begin{array}{ccccc}
0 & 1 & 0 & \cdots & 0 \\
0 & 0 & 1 & \cdots & 0 \\
\vdots & \vdots & & & 0 \\
-A_{0}(r) & -A_{1}(r) & -A_{2}(r) & \cdots & -A_{n-1}(r)
\end{array}\right) \mathbf{x} \\
& +\mathbf{B} S=\mathbf{A}_{\mathbf{c}} \mathbf{x}+\mathbf{B} S .
\end{aligned}
$$


Thus, the closed-loop characteristic polynomial is given by

$$
\begin{aligned}
f_{r}(t) & =t^{n}+A_{n-1}(r) t^{n-1}+\cdots+A_{0}(r) \\
& =t^{n}+\frac{p^{n-1}(-r)}{(n-1) !} t^{n-1}+\cdots+\frac{p(-r)}{0 !} \\
& =p_{A}(t-r) .
\end{aligned}
$$

When $r=0, A_{0}$ is a stable matrix and $f_{0}(t)=p_{A}(t)$ but when $r>-\sigma_{p_{A}}$ we can obtain dissipative systems with unstable dynamics and the possibility of generating multiscroll attractors. As described in Definition 21, a system with stability index $n-2$ will be addressed as a system of the UDS type I. Besides, the following considerations have to be made in order to call (25) a UDS of type I that in addition generates an attractor $\mathfrak{A}$.

(a) The linear part of the system must satisfy the dissipative condition $\sum_{i=1}^{n} \lambda_{i}<0$, where $\lambda_{i}, i=1,2, \ldots, n$, are eigenvalues of $\mathbf{A}_{\mathbf{c}}$. Consider also that $n-2$ eigenvalues are negative real numbers, and two $\lambda_{i}$ values are complex conjugate eigenvalues with positive real part $\operatorname{Re}\left\{\lambda_{i}\right\}>0$, resulting in an unstable focus-saddle equilibrium $\mathbf{X}^{*}$. This type of equilibria presents a stable manifold $M^{s}=\operatorname{span}\left\{V_{\lambda_{1}}, \ldots, \lambda_{n-2}\right\} \in \mathbb{R}^{n}$ with a fast eigendirection and an unstable manifold $M^{u}=$ $\operatorname{span}\left\{V_{\lambda_{n-1}}, V_{\lambda_{n}}\right\} \in \mathbb{R}^{n}$ with a slow spiral eigendirection, where $V_{\lambda_{i}}$ corresponds to the eigenvector of $\mathbf{A}$ regarding the eigenvalue $\lambda_{i}$.

(b) The affine vector BS must be considered as a discrete function that changes depending on which domain $\mathscr{D}_{i} \subset \mathbb{R}^{n}$ the trajectory is located at. Accordingly $\mathbb{R}^{n}=\bigcup_{i=1}^{k} \mathscr{D}_{i}$. Then a switching system based on (25) is given by

$$
\begin{gathered}
\dot{\mathbf{X}}=\mathbf{A}_{\mathbf{c}} \mathbf{X}+\mathbf{B S}(\mathbf{X}) \\
\mathbf{S}(\mathbf{X})= \begin{cases}s_{1}, & \text { if } X \in \mathscr{D}_{1} ; \\
s_{2}, & \text { if } X \in \mathscr{D}_{2} ; \\
\vdots & \vdots \\
s_{k}, & \text { if } X \in \mathscr{D}_{k} .\end{cases}
\end{gathered}
$$

The equilibria of system (29) are $\mathbf{X}_{i}^{*}=-\mathbf{A}_{\mathbf{c}}{ }^{-1} \mathbf{B S}$, with $i=1, \ldots, k$, and each entry $s_{i}$ of the switching system is considered in order to preserve bounded trajectories of system (29). Thence, the choice of $c_{i}$ 's in the definition of the step function $\mathbf{S}$ will determine the commutation regions $\mathscr{D}_{i}$ 's that enclose each equilibrium $\mathbf{X}_{i}^{*}$.

The commuting system given by (29) induces in phase space $\mathbb{R}^{n}$ the flow $\left(\varphi^{t}\right), t \in \mathbb{R}$, such that each forward trajectory of the initial point $\mathbf{X}_{0}=\mathbf{X}(t=0)$ is the set $\{\mathbf{X}(t)=$ $\left.\varphi^{t}\left(\mathbf{X}_{0}\right): t \geq 0\right\}$. Furthermore, these systems have a dissipative bounded region $\Omega \subset \mathbb{R}^{n}$ named basin of attraction, such that the flow $\varphi^{t}(\Omega) \subset \Omega$ for every $t \geq 0$. The attractor $\mathfrak{A}$ is the largest attracting invariant subset of $\Omega$.
Definition 25. Consider a system given by (29) in $\mathbb{R}^{n}$ and equilibrium points $\mathbf{X}_{i}^{*}$, with $i=1, \ldots, k$ and $k \geq 2$. We say that system (29) can generate multiscroll attractors with the minimum of equilibrium points, if for any initial condition $X_{0} \in \mathfrak{B} \subset \mathbb{R}^{n}$ in the basin of attraction the orbit $\varphi\left(X_{0}\right)$ generates an attractor $\mathfrak{A} \subset \mathbb{R}^{n}$ with oscillations around each $\mathbf{X}_{k}^{*}$.

We exemplify the theory by presenting a case in $\mathbb{R}^{3}$ where the following theorem holds.

Theorem 26. Consider system (25) for the particular case where the dimension is three. That is, consider a 3D-control system with characteristic Hurwitz polynomial $p_{A}(t)=(t+$ $\zeta)(t+\bar{\zeta})(t+\rho)$, where $\operatorname{Im}(\zeta) \neq 0$. If $\operatorname{Re}(\zeta) \neq \rho$, then the closed-loop system with the control $u=c^{T}(r) x$ is UDS for all $r \in\left(-\sigma_{p_{A}}, U_{\operatorname{diss}\left(p_{A}\right)}\right)$.

Proof. Note that the closed-loop system (25) with the feedback $u=c^{T}(r) x$ has a characteristic polynomial to the polynomial family $f_{r}(t)=p_{A}(t-r)$. Then by Corollary $24 f_{r}(t)$ is UDS for all $r \in\left(-\sigma_{p_{A}}, U_{\operatorname{diss}\left(p_{A}\right)}\right)$. This completes the proof.

A system satisfying the previous theorem is candidate to generate multiscroll attractors emerging from its equilibria with a suitable step function $S$. The number of scrolls in the attractor $\mathfrak{A}$ is due to the step function $S$. Next, let us illustrate the generation of multiscroll attractors. Consider the system

$$
\dot{x}=\left(\begin{array}{ccc}
0 & 1 & 0 \\
0 & 0 & 1 \\
-50 & -20 & -7
\end{array}\right) x+\left(\begin{array}{c}
0 \\
0 \\
7.0278
\end{array}\right) S+\left(\begin{array}{l}
0 \\
0 \\
1
\end{array}\right) u
$$

with step function

$$
\begin{aligned}
& S\left(x_{1}\right)= \begin{cases}3, & \text { for } 0.5<x_{1} \\
2, & \text { for } 0.3<x_{1} \leq 0.5 \\
1, & \text { for } 0.1<x_{1} \leq 0.3 \\
0, & \text { for } x_{1} \leq 0.1\end{cases} \\
& u=\left(50-p(-r), 20-p^{\prime}(-r) / 1 !, 7-p^{\prime \prime}(-r) / 2 !\right) x \text {, where } p(t)= \\
& t^{3}+7 t^{2}+20 t+50 \text { is Hurwitz. } \\
& \dot{x}=\left(\begin{array}{ccc}
0 & 1 & 0 \\
0 & 0 & 1 \\
-p(-r) & -\frac{-p^{\prime}(-r)}{1 !} & -\frac{-p^{\prime \prime}(-r)}{2 !}
\end{array}\right) x \\
& +\left(\begin{array}{c}
0 \\
0 \\
7.0278
\end{array}\right) S
\end{aligned}
$$

Denote $f_{r}(t)=t^{3}+\left(p^{\prime \prime}(-r) / 2 !\right) t^{2}+\left(p^{\prime}(-r) / 1 !\right) t+p(-r)$ for $r=0 . f_{0}(t)=p(t)=t^{3}+7 t^{2}+20 t+50$ is a Hurwitz polynomial 


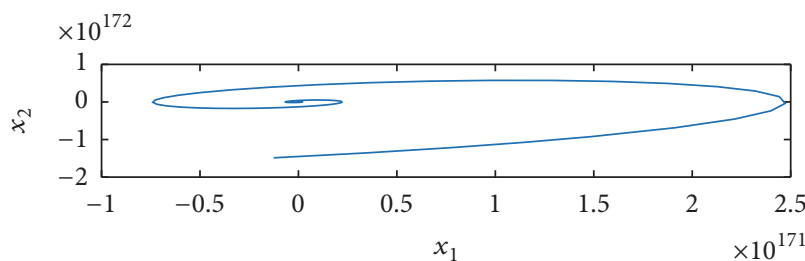

(a)

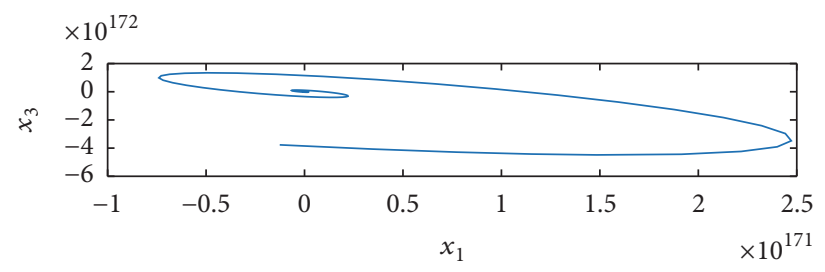

(b)

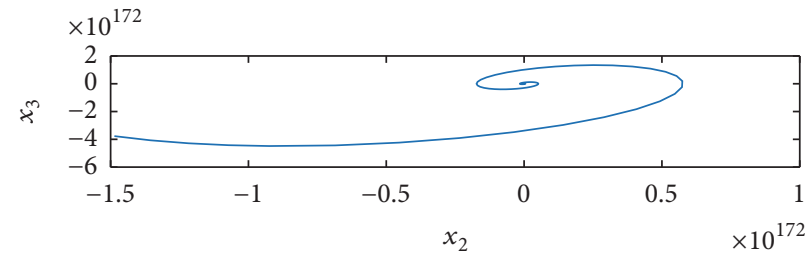

(c)

Figure 1: Projections of the solution of system (30) onto the planes: (a) $\left(x_{1}, x_{2}\right)$; (b) $\left(x_{1}, x_{3}\right)$; and (c) $\left(x_{2}, x_{3}\right)$.

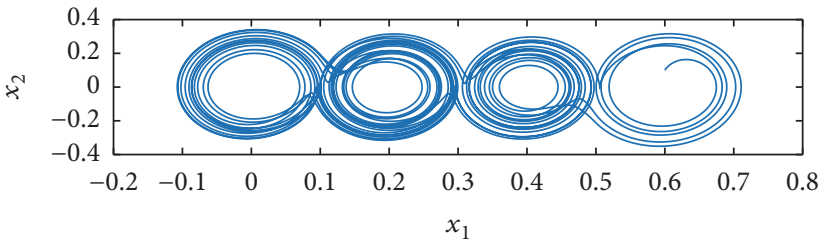

(a)

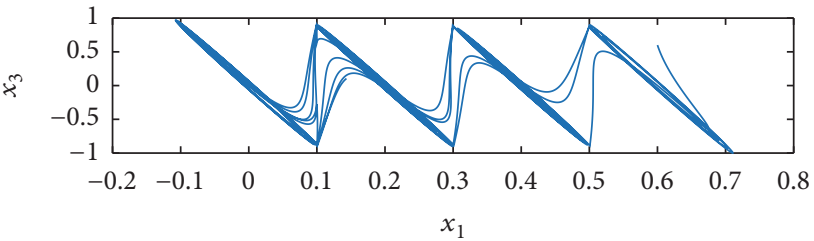

(b)

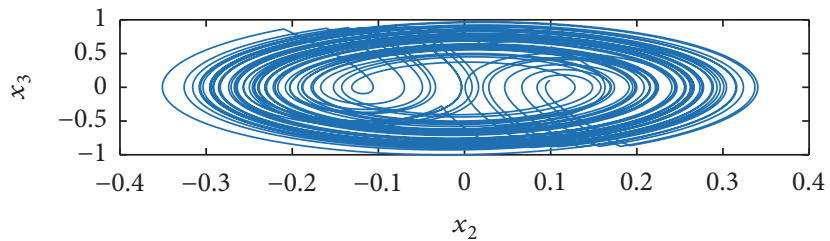

(c)

Figure 2: Projections of the attractor onto the planes: (a) $\left(x_{1}, x_{2}\right)$; (b) $\left(x_{1}, x_{3}\right)$; and (c) $\left(x_{2}, x_{3}\right)$.

and there is no multiscroll. Figure 1 shows the projection of the stable solution onto the planes: (a) $\left(x_{1}, x_{2}\right)$; (b) $\left(x_{1}, x_{3}\right)$; and $(\mathrm{c})\left(x_{2}, x_{3}\right)$.

The abscissa of $f_{0}(t)$ is $\sigma_{f_{0}}=-1$. Then other behavior could appear when $r \in(1, \infty)$. For example, for $r=1.1$, $f_{2}(t)=t^{3}+3.7 t^{2}+8.23 t+35.139$; hence $\sum_{j=1}^{3} t_{j}<0$; consequently system (32) is dissipative when $r=1.1$ and in Figure 2 the generation of multiscroll attractor is illustrated. Another reference where multiscroll attractors have been studied is [40].

The equilibria of the system for $r=1.1$ are given by $\mathbf{X}_{1}^{*}=(0.6,0,0)^{T}, \mathbf{X}_{2}^{*}=(0.4,0,0)^{T}, \mathbf{X}_{3}^{*}=(0.2,0,0)^{T}$, and $\mathbf{X}_{4}^{*}=(0,0,0)^{T}$. Between equilibria, the commutation surfaces at the planes are as follows: $P_{i}=\left\{\left(x_{1}, x_{2}, x_{3}\right)^{T} \in \mathbb{R}^{3} \mid\right.$ $\left.x_{1}=0.1+0.2 *(i-1)\right\}$, with $i=1,2,3$, dividing the space into four domains $\mathscr{D}_{1,2,3,4}$ given by $\mathscr{D}_{1}=\left\{\left(x_{1}, x_{2}, x_{3}\right)^{T} \in \mathbb{R}^{3}\right.$ | $\left.0.5<x_{1}\right\}, \mathscr{D}_{2}=\left\{\left(x_{1}, x_{2}, x_{3}\right)^{T} \in \mathbb{R}^{3} \mid 0.3<x_{1} \leq 0.5\right\}$, $\mathscr{D}_{3}=\left\{\left(x_{1}, x_{2}, x_{3}\right)^{T} \in \mathbb{R}^{3} \mid 0.1<x_{1} \leq 0.3\right\}$, and $\mathscr{D}_{4}=$ $\left\{\left(x_{1}, x_{2}, x_{3}\right)^{T} \in \mathbb{R}^{3} \mid x_{1} \leq 0.1\right\}$. Notice two important facts about the system; first the scrolls are increasing their size due to the unstable manifold; this can be better appreciated at the projection of the attractor onto the $\left(x_{1}, x_{3}\right)$ plane from Figure 2(b). Second, the trajectory of the system oscillating around the equilibrium point $\mathbf{X}_{4}^{*}$ in $\mathfrak{A} \cap \mathscr{D}_{4}$ escapes from the domain $\mathscr{D}_{4}$ located in the left side of the commutation surface. This occurs near the unstable manifold $E^{u} \subset \mathscr{D}_{4}$ where it crosses the commutation surface and it is attracted by the stable manifold $E^{\mathcal{S}} \subset \mathscr{D}_{3}$ to the equilibrium point $\mathbf{X}_{3}^{*}$ in the domain $\mathscr{D}_{3}$ located at the right side of the commutation surface $P_{1}$. The process is repeated in the inverse way forming scrolls around each equilibrium point.

\section{Conclusion}

In this paper we use the Gauss-Lucas theorem for obtaining an inequality between the abscissas of stability of a Hurwitz polynomial and its derivative. Then we use such inequality for getting a lower bound for the abscissa of a polynomial and for an interval family of polynomials. We have compared the lower bounds obtained with other works and we can say that the obtained bounds in this paper are easy to 
calculate and sometimes are better that others. Based on the aforementioned results, an approach to generate multiscroll attractors was presented. We consider that this result is important to help in understanding the emergence of chaos in stable systems. Using the abscissa of stability we can generate multiscroll attractors from a Hurwitz polynomial. One interesting aspect is that we can generate multiscroll attractor with the change of only one parameter.

\section{Conflicts of Interest}

The authors declare that there are no conflicts of interest regarding the publication of this paper.

\section{Acknowledgments}

The first author wishes to thank CONACYT for its Ph.D. scholarship support. Jorge Antonio López-Rentería also wishes to thank CONACYT for the postdoctoral grant (Grant no. 290941-UIA) and the Iberoamerican University for the support in the realization of this paper. C. A. LoredoVillalobos also wishes to acknowledge the support of CONACYT through the postdoctoral fellowship. Eric CamposCantón acknowledges the CONACYT financial support for sabbatical. He would also like to thank the University of Houston for his sabbatical support and Professor Matthew Nicol for allowing him to work with him and his valuable discussions on dynamic systems.

\section{References}

[1] J. C. Maxwell, “On Governors," Proceedings of the Royal Society of London, vol. 16, pp. 270-283, 1868.

[2] A. Hurwitz, "Ueber die Bedingungen, unter welchen eine Gleichung nur Wurzeln mit negativen reellen Theilen besitzt," Mathematische Annalen, vol. 46, no. 2, pp. 273-284, 1895.

[3] S. P. Bhattacharyya, H. Chapellat, and L. H. Keel, Robust Control: The parametric approach, Prentice-Hall, Upper Saddle River, NJ, USA, 1995.

[4] F. R. Gantmacher, The Theory of Matrices, vol. 1, Chelsea Publishing, New York, NY, USA, 1959.

[5] P. Lancaster and M. Tismenetsky, The Theory of Matrices, Academic Press, New York, NY, USA, 2nd edition, 1985.

[6] M. Marden, Geometry of polynomials, Mathematical Surveys, No. 3, American Mathematical Society, Providence, R.I., USA, 2nd edition, 1966.

[7] V. L. Kharitonov, "Asymptotic stability of an equilibrium position of a family of systems of linear differential equations," Differentsial'nye Uravneniya, vol. 14, pp. 2086-2088, 1978.

[8] F. Blanchini, R. Tempo, and F. Dabbene, "Computation of the minimum destabilizing volume for interval and affine families of polynomials," Institute of Electrical and Electronics Engineers. Transactions on Automatic Control, vol. 43, no. 8, pp. 1159-1163, 1998.

[9] F. Dabbene, B. T. Polyak, and R. Tempo, "On the complete instability of interval polynomials," Systems \& Control Letters, vol. 56, no. 6, pp. 431-438, 2007.

[10] A. A. Kale and A. L. Tits, "On Kharitonov's theorem without invariant degree assumption," Automatica. A Journal of IFAC, the International Federation of Automatic Control, vol. 36, no. 7, pp. 1075-1076, 2000.

[11] R. Tempo, “A dual result to Kharitonov's theorem," Institute of Electrical and Electronics Engineers. Transactions on Automatic Control, vol. 35, no. 2, pp. 195-198, 1990.

[12] J. C. Willems and R. Tempo, "The Kharitonov theorem with degree drop," Institute of Electrical and Electronics Engineers. Transactions on Automatic Control, vol. 44, no. 11, pp. 22182220, 1999.

[13] B. Aguirre, C. Ibarra, and R. Suárez, "Sufficient algebraic conditions for stability of cones of polynomials," Systems \& Control Letters, vol. 46, no. 4, pp. 255-263, 2002.

[14] B. Aguirre and R. Suárez, "Algebraic test for the Hurwitz stability of a given segment of polynomials," Boletín de la Sociedad Matemática Mexicana 1 No. 2, vol. 12, no. 2, pp. 261275, 2006.

[15] S. Bialas, "A necessary and sufficient condition for the stability of convex combinations of stable polynomials or matrices," Bulletin of the Polish Academy of Sciences. Technical Sciences, vol. 33, no. 9-10, pp. 473-480, 1985.

[16] J.-A. López-Renteria, B. Aguirre-Hernández, and F. Verduzco, "The boundary crossing theorem and the maximal stability interval," Mathematical Problems in Engineering, Article ID 123403, 13 pages, 2011.

[17] J. Ackerman, Robust Control. The Parameter Space Approach, Springer-Verlag, New York, 2002.

[18] R. B. Barmish, New Tools for Robustness of Linear Systems, MacMillan Publishing Co, New York, 1994.

[19] P. Dorato, R. Tempo, and G. Muscato, "Bibliography on robust control," Automatica. A Journal of IFAC, the International Federation of Automatic Control, vol. 29, no. 1, pp. 201-213, 1993.

[20] O. Taussky-Todd, "On stable matrices," in Colloques Internationaux Du Centre National De La Recherche Scientifique, vol. 165, pp. 75-88, Paris, France, 1968.

[21] V. Zakian and U. Al-Naib, "Design of dynamical and control systems by the method of inequalities," Proceedings of the Institution of Electrical Engineers, vol. 120, no. 11, pp. 1421-1427, 1973.

[22] V. Zakian, "New formulation for the method of inequalities," Proceedings of the Institution of Electrical Engineers, vol. 126, no. 6, pp. 579-584, 1979.

[23] G. Schrack F, Lower Bounds for the Abscissa of Stability of Stable Polynomials. Dissertation 4065 [M.sc. thesis], Eidgenossische Technische Hochschule, Zurich, 1967.

[24] G. F. Schrack, "Lower bounds to the abscissa of stability of a stable polynomial from symmetric functions," SIAM Journal on Applied Mathematics, vol. 21, pp. 373-379, 1971.

[25] S. Bialas, "Upper bounds for the abscissa of stability of a stable interval polynomial," Bulletin of the Polish Academy of Sciences. Mathematics, vol. 32, no. 1-2, pp. 1-9, 1984.

[26] P. Henrici, "Upper bounds for the abscissa of stability of a stable polynomial," SIAM Journal on Numerical Analysis, vol. 7, pp. 538-544, 1970.

[27] K. L. Olifirov, "Determination of a neighborhood of the imaginary axis which is disjoint from the spectrum of a real polynomial," Mathematical Notes of the Academy of Sciences of the USSR, vol. 22, no. 2, pp. 581-584, 1977.

[28] E. Campos-Cantón, J. G. Barajas-Ramírez, G. Solís-Perales, and R. Femat, "Multiscroll attractors by switching systems.," Chaos (Woodbury, N.Y.), vol. 20, no. 1, p. 013116, 2010. 
[29] E. Campos-Cantón, R. Femat, and G. Chen, "Attractors generated from switching unstable dissipative systems," Chaos, vol. 22, no. 3, Article ID 033121, 2012.

[30] J. Lü, F. Han, X. Yu, and G. Chen, "Generation 3-D multiscroll chaotic attractors: a hysteresis series switching methods," Automatica, vol. 40, no. 10, pp. 1677-1687, 2014.

[31] L. J. Ontanon-García, E. Jimenez-López, E. Campos-Cantón, and M. Basin, "A family of hyperchaotic multi-scroll attractors in Rn," Applied Mathematics and Computation, vol. 233, pp. 522533, 2014

[32] C. Sánchez-López, R. Trejo-Guerra, J. M. Muñoz-Pacheco, and E. Tlelo-Cuautle, "N-scroll chaotic attractors from saturated function series employing CCII+s," Nonlinear Dynamics, vol. 61, no. 1-2, pp. 331-341, 2010.

[33] J. A. K. Suykens and J. Vandewalle, "Generation of n-Double Scrolls $(\mathrm{n}=1,2,3,4, \ldots)$," IEEE Transactions on Circuits and Systems I: Fundamental Theory and Applications, vol. 40, no. 11, pp. 861-867, 1993.

[34] J. A. K. Suykens, A. Huang, and L. O. Chua, "A family of n-scrolls attractors from a generalized Chuas circuit," International Journal of Electronics and Communications, vol. 51, no. 3, pp. 131-138, 1997.

[35] M. s. Yalcin, J. A. Suykens, J. Vandewalle, and S. Özoguz, "Families of scroll grid attractors," International Journal of Bifurcation and Chaos in Applied Sciences and Engineering, vol. 12, no. 1, pp. 23-41, 2002.

[36] H. S. Wilf, Mathematics for the physical sciences, Dover Publications, Inc., 180 Varick Street, New York, NY, USA, 1978.

[37] V. Zakian, "Computation of the abscissa of stability by repeated use of the Routh test," Institute of Electrical and Electronics Engineers. Transactions on Automatic Control, vol. 24, no. 4, pp. 604-607, 1979.

[38] V. Zakian, "A criterion of approximation for the method of inequalities," International Journal of Control, vol. 37, no. 5, pp. 1103-1111, 1983.

[39] V. Zakian, Control Systems Design: A New Framework, Springer-Verlag, London, UK, 2005.

[40] B. Aguirre-Hernández, E. Campos-Cantón, J. A. LópezRenteria, and E. C. Díaz González, "A polynomial approach for generating a monoparametric family of chaotic attractors via switched linear systems," Chaos, Solitons \& Fractals, vol. 71, pp. 100-106, 2015.

[41] E. C. Díaz-González, J.-A. López-Rentería, E. Campos-Cantón, and B. Aguirre-Hernández, "Maximal unstable dissipative interval to preserve multi-scroll attractors via multi-saturated functions," Journal of Nonlinear Science, vol. 26, no. 6, pp. 18331850, 2016. 


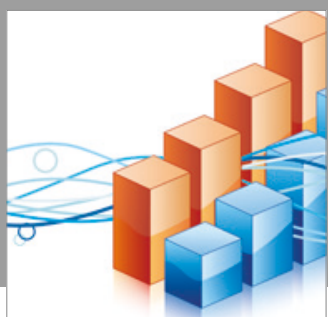

Advances in

Operations Research

vatersals

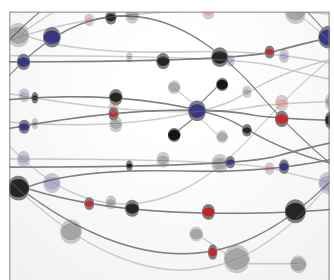

\section{The Scientific} World Journal
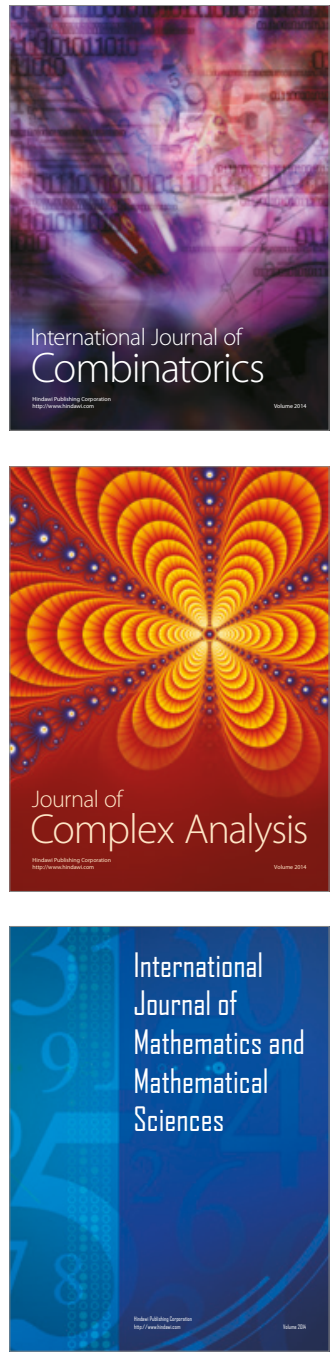
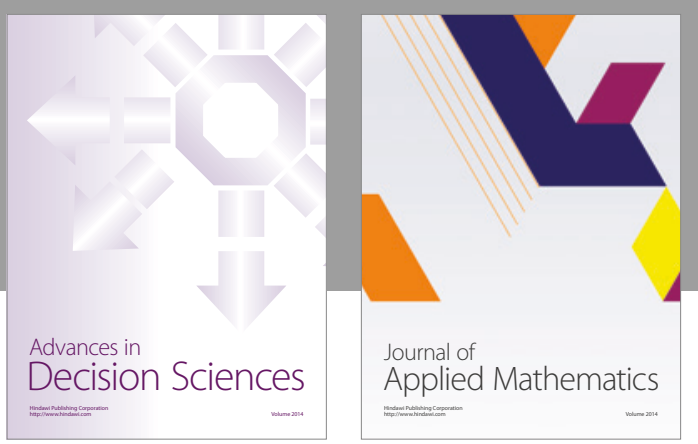

Algebra

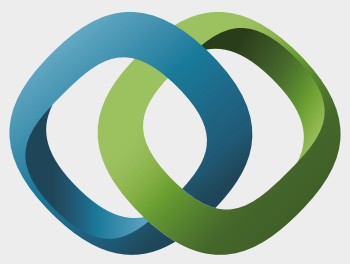

\section{Hindawi}

Submit your manuscripts at

https://www.hindawi.com
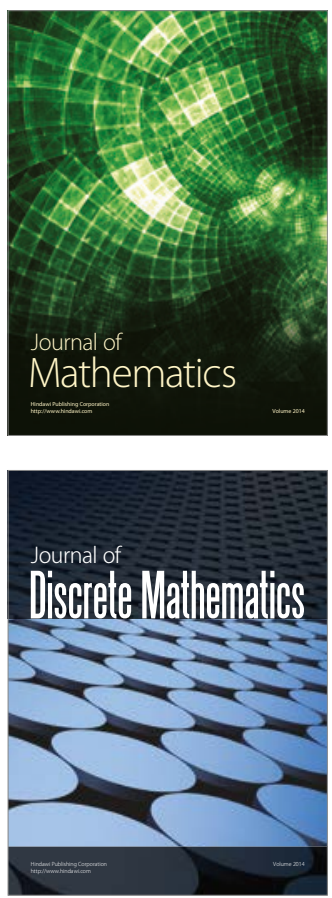

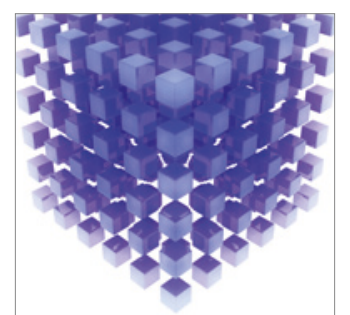

Mathematical Problems in Engineering
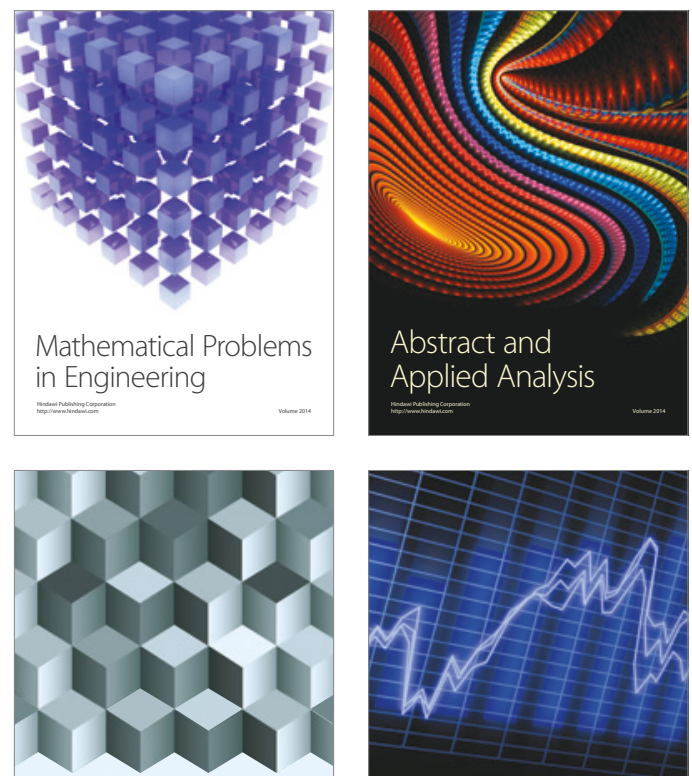

Journal of

Function Spaces

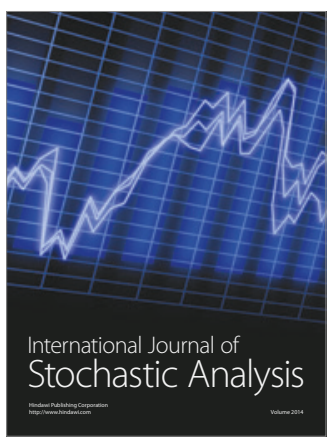

Probability and Statistics
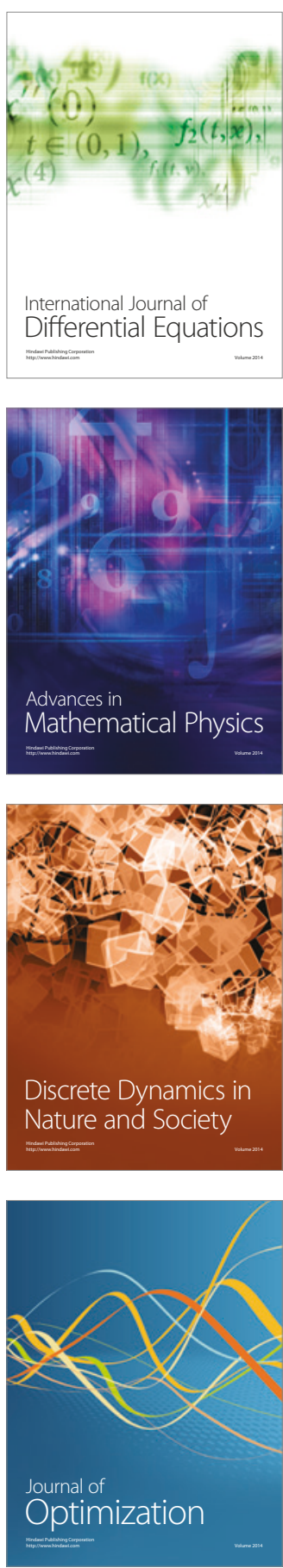